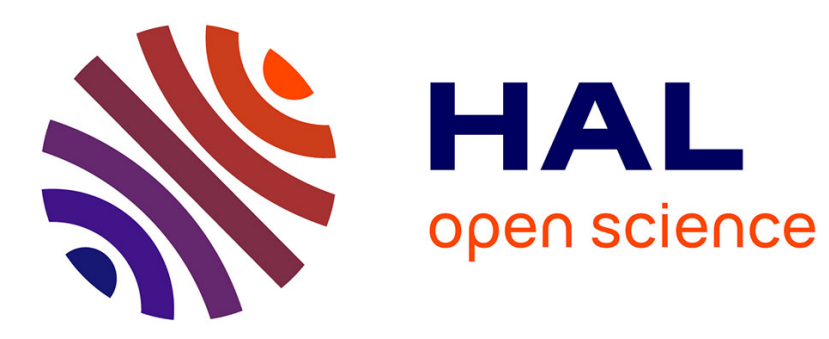

\title{
New Nanocomposite Materials
}

B. Hamdoun, D. Ausserré, S. Joly, Y. Gallot, V. Cabuil, C. Clinard

\section{To cite this version:}

B. Hamdoun, D. Ausserré, S. Joly, Y. Gallot, V. Cabuil, et al.. New Nanocomposite Materials. Journal de Physique II, 1996, 6 (4), pp.493-501. 10.1051/jp2:1996106 . jpa-00248311

\section{HAL Id: jpa-00248311 https://hal.science/jpa-00248311}

Submitted on 1 Jan 1996

HAL is a multi-disciplinary open access archive for the deposit and dissemination of scientific research documents, whether they are published or not. The documents may come from teaching and research institutions in France or abroad, or from public or private research centers.
L'archive ouverte pluridisciplinaire HAL, est destinée au dépôt et à la diffusion de documents scientifiques de niveau recherche, publiés ou non, émanant des établissements d'enseignement et de recherche français ou étrangers, des laboratoires publics ou privés. 


\title{
New Nanocomposite Materials
}

B. Hamdoun $\left({ }^{1}\right)$, D. Ausserré $\left({ }^{1, *}\right)$, S. Joly $\left({ }^{1}\right)$, Y. Gallot $\left({ }^{2}\right)$, V. Cabuil $\left({ }^{3}\right)$ and C. Clinard $\left({ }^{4}\right)$

(1) Laboratoire de Physique de l'État Condensé $\left({ }^{* *}\right)$, Université du Maine, BP 535, 72017 Le Mans Cedex, France

$\left({ }^{2}\right)$ Institut Charles Sadron (Centre de Recherche Macromoléculaire-École des Hauts Polymères), 6 rue Boussingault, 67083 Strasbourg Cedex, France

(3) Laboratoire de Physico-Chimie Inorganique, Université P. \& M. Curie, Tour 54, 75252 Paris Cedex 05, France

$\left({ }^{4}\right)$ Centre de Nanoscopie Électronique Analytique, Université d'Orléans, BP 6759, rue de Chartres, 45067 Orléans Cedex, France

(Received 18 September 1995, revised 13 December 1995, accepted 19 February 1996)

PACS.05.90.+m - Other topics in statistical physics and thermodynamics

PACS.64.75 +g - Solubility, segregation, and mixing; phase separation

PACS.61.25.Hq - Macromolecular and polymer solutions; polymer melts; swelling

\begin{abstract}
The first successful elaboration of a new class of thermodynamically stable nano composite materials is reported. These were obtained in three steps: 1) mixing of solutions of large diblock copolymers and of modified mineral nanoparticles; 2) spincoating the composite solution on a solid substrate; 3 ) annealing the resulting film till quasi-equilibrium self organization is reached. A composite organic-inorganic material is obtained, with a periodic distribution of the particles in the polymeric matrix.
\end{abstract}

\section{Introduction}

Diblock copolymers A/B are strongly asymmetric macromolecules made of two unlike homopolymers linked together by a covalent bond. They are known to self organize in the melt state into a regular array of A-rich and B-rich microdomains [1]; these can be sheets organized in lamellar stacking, cylinders on an hexagonal lattice, spheres on a cubic lattice, or connected arrangements with cubic or more exotic symmetries. The main parameter which controls the texture is the relative length of the two subchains. Self-assembly is driven by the immiscibility of the two chemical components. Upon phase separation, the system minimizes the sum of the interfacial energy associated to A-B contacts and of the elastic free energy associated to normal stretching of the chains. Thin films of so-called "symmetric" diblock copolymers, i.e. with the same numbers $N_{\mathrm{A}}$ and $N_{\mathrm{B}}$ of monomers along each subchain can be prepared on solid substrates [2]. Self-organization then leads to a lamellar stacking often with homeotropic boundary conditions. Minimization of surface energy at the boundaries dictates which block

$\left({ }^{*}\right)$ Author for correspondence

$\left({ }^{* *}\right)$ URA CNRS $N^{\circ} 807$

(C) Les Éditions de Physique 1996 
is present at the air/polymer and substrate/polymer interfaces. The lamellar period $L$ corresponds to the thickness of an ABBA bilayer. In practice, these smectic films are made by spincoating a copolymer solution on the solid substrate and annealing the subsequent solid film at a temperature which is intermediate between the highest glass transition temperature of the two components, $T_{\mathrm{g}}$, and the order-disorder transition temperature, $T_{\text {od }}$, at which $\mathrm{A}$ and $\mathrm{B}$ bulk microdomains disappear.

The initial idea of the present work is the following: imagine very small solid or liquid particles embedded in the self organized medium with a strong affinity for one of the two polymeric species, A for instance. The particles will tend to accumulate into the A-rich domains. Thus their spatial distribution will reproduce the ordered arrangement of the copolymer mesophase and be periodically modulated or even crystalline, with a very large mesh size when compared to particle dimensions. The macroscopic properties of the composite will now reflect both the physical properties which are specific of nanoobjects, such as ability to form ferromagnetic monodomains or optical microcavities or to generate third order harmonic optical waves, and those which are characteristic of large mesh periodic structure, the most obvious being a possible coherent response to electromagnetic radiation. The potential interest of such materials has been recognized for a while and successful attempts to build copolymer/nanoparticle composite organized structures have been reported already. These fall into three categories: i) metallic complexes are attached on the A backbone prior to self organization. Once the ordering is achieved, metal atoms are released from the backbone by thermal or electromagnetic activation and aggregate into small clusters, the size of which is controlled by the duration of the process [3]; ii) metallic species are introduced via a solvent in the previously self-organized copolymer microphase where they react with functionalized parts of the macromolecules, and temporarily stay on the chains, with the possibility of being released afterwards, as in the preceding case $[4,5]$; iii) reactive compounds are added to the copolymer structure; after self organization, the chemical reaction is externally activated and the additives form the nanoparticles [6]. In all these techniques, the clusters form in situ after the matrix is organized. They are selectively embedded in polymer A as demanded, but clearly none of these methods provides a good control of the cluster structure.

On the contrary our route decouples the nanoparticle synthesis and the composite organization, which allows us to choose a large range of nanoparticle properties. It consists in first preparing nanoparticles with the desired features. Their surface state is then modified in such a way that, on the one hand they become soluble in a solvent of the copolymer, and, on the other hand, they can dissolve in a melt of $\mathrm{A}$ but not in a melt of $\mathrm{B}$. The most straightforward way to achieve this is to cover the particles with a grafted brush of $A$ polymer chains. In the next step, a solution of both copolymer chains and modified nanoparticles is prepared in a common solvent and the solution is spincoated on a smooth solid substrate. Further annealing at a temperature $T>T_{\mathrm{g}}$ leads to spontaneous ordering of the mixture. The resulting structure of the composite material is thermodynamically stable.

The validity of this method will now be illustrated by a specific example: namely, that of ferrofluid nanoparticles embedded in a symmetric diblock copolymer. It has been found that for an adequate choice of the grafted species (A or B) and for a ratio of particle size to polymer block length sufficiently small, the composite structure remains very similar to that of a pure copolymer film. Some lamellar ordering is actually conserved up to nanoparticle volume fractions of about $25 \%$.

Homeotropic alignment of the microdomains makes it possible to probe the composite film by absorption TEM on thin slides which are cut normally to the free surface. Moreover, ordered symmetric copolymer thin films are known to exhibit characteristic reliefs with holes and islands due to the quantization of their thickness [7]. Since their observation is straightforward, it was 
useful to establish correlations between internal structure and relief geometry. As a result, evidence of nanoparticle inclusion can now be obtained on these systems by simple surface probes. This will be illustrated in what follows.

The detailed nature of the various components and the main features of the characterization techniques will be briefly described in the experimental section of this paper. Results will be reported and discussed in the following section.

\section{Experimental}

The diblock copolymer is a polystyrene-polybutylmethacrylate $\mathrm{P}$ (S-b-PBMA). The symmetric sample has a molecular weight $M_{\mathrm{w}}=82000$ and a polydispersity index $M_{\mathrm{w}} / M_{\mathrm{n}}=1.05$. The weight fraction of PS is 0.526 . Nanoparticles are made of maghemite $\left(\gamma-\mathrm{Fe}_{2} \mathrm{O}_{3}\right)$, a wellknown ferrofluid. Their average size is $3.5 \mathrm{~nm}$ with a large distribution $(\sigma=0.4)$, which implies an intrinsic superparamagnetic behaviour [8]. They are covered by a PS layer of short chains $\left(M_{\mathrm{w}}=13 \mathrm{~K}\right)$ using the procedure described in reference [9]. For connection with previous notations, PS blocks correspond to species A. Stable solutions of the copolymer and the nanoparticles were separately prepared in toluene with the same volume fraction of $0.01 \%$. The two solutions were then mixed in the required proportions prior to spin coating.

For the transmission electron microscopy (TEM) studies, organic substrates with a low surface roughness were used (Orma lenses, Essilor, France, rms $\approx 5 \AA$ ). Composite films were annealed under vacuum for 48 hours at $T=150^{\circ} \mathrm{C}$. Microtomed sections were obtained using a Reichert ultra microtome equipped with a diamond knife. The microscope is a Philipps CM 20 apparatus operating at $200 \mathrm{KV}$. Contrast between the copolymer components was obtained by $\mathrm{RuO}_{4}$ staining of the PS phase [10]. The contrast was adjusted so that PS appears darker than PBMA and the nanoparticles appear darker than the PS domains in which they are located. Atomic force microscopy (AFM) studies were carried out on a Dimension 3000 nanoscope (Digital) working in the tapping mode. Optical micrography was performed on a Reichert Polyvar Microscope working in the reflecting mode.

\section{Results and Discussion}

Figure 1a presents a TEM cross section of a lamellar composite with a nanoparticle volume fraction of about $25 \%$ in the symmetric diblock. The grey areas of the micrographs are segregated PS sequences labelled with $\mathrm{RuO}_{4}$, the white areas are PBMA phases and the black spots are made of colloidal particles. The value of the lamellar period $L$ is $32 \mathrm{~nm}$. This estimation was first made from TEM pictures and was later corroborated and refined by the AFM studies which are evoked below. It is significantly larger than that of the pure copolymer system, estimated to $30 \mathrm{~nm}$ using AFM. However, the relative difference $\Delta L / L$ is also significantly smaller than the nanoparticle volume fraction. This contradicts an a priori reasonable scenario in which the nanoparticles would localize at the frontier between two interpenetrating $A$ brushes, leaving the elastic free energy of A chains practically unchanged. The observed deviation from that expectation implies a significative change of the interfacial area per molecule, $\Sigma_{c}$, which implies an additional stretching of the polymer. It clearly demonstrates that the chains have to find a new equilibrium state in the presence of the embedded particles. It is also consistent with the fact that the black regions corresponding to high concentrations in nanoparticles (unfortunately unresolved) seem to be distributed quite homogeneously through the A microdomains rather than aggregated in the middle. The last point to be mentioned about Figure 1a concerns the roughness of the A/B interface. It is much higher than what is observed with the pure copolymer, Figure 1b, and furthermore the position of the lamellae fluctuates much more. 


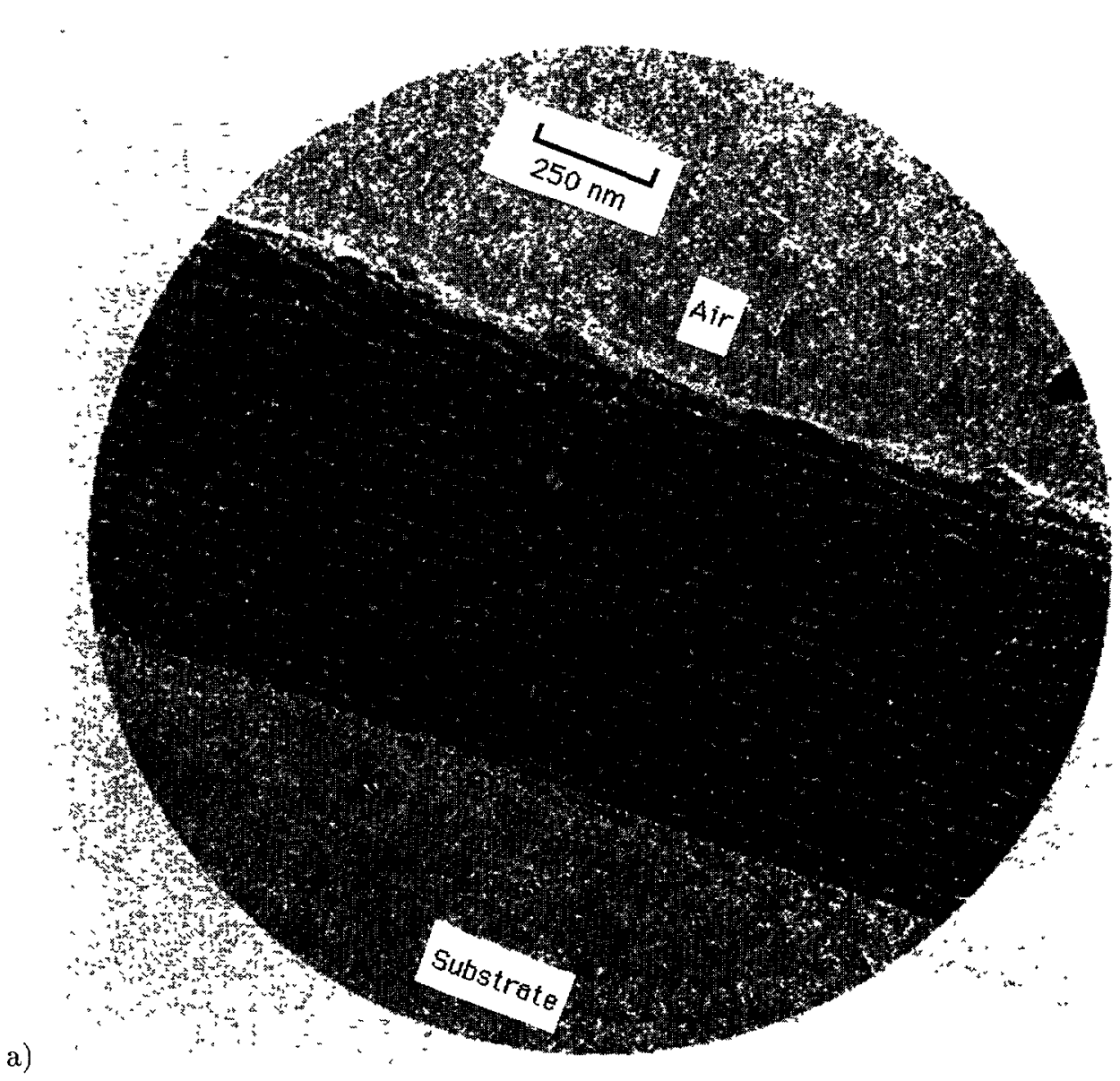

a)

$150 \mathrm{~nm}$

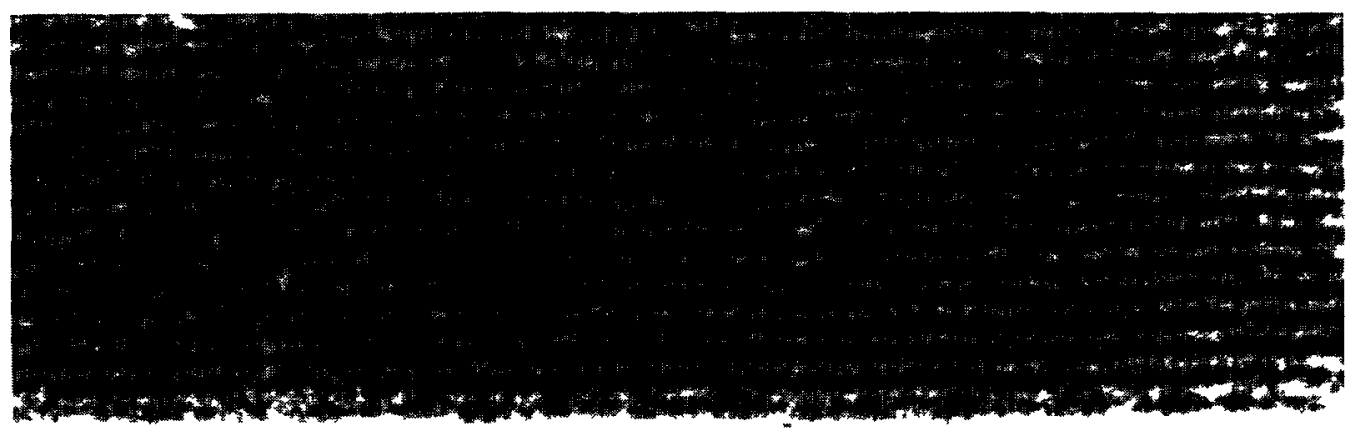

$\left.a^{3}\right)$

Substrate

Fig. 1. - a) TEM micrograph of the cross-section of a lamellar composite film with a 25 per cent volume fraction of nanoparticles. A polymer layer is grafted on the particles. Particle diameter $=$ $3.5 \mathrm{~nm} . \mathrm{a}^{\prime}$ ) is an enlargement. b) TEM micrograph of the cross-section of a lamellar pure copolymer film. 
b)

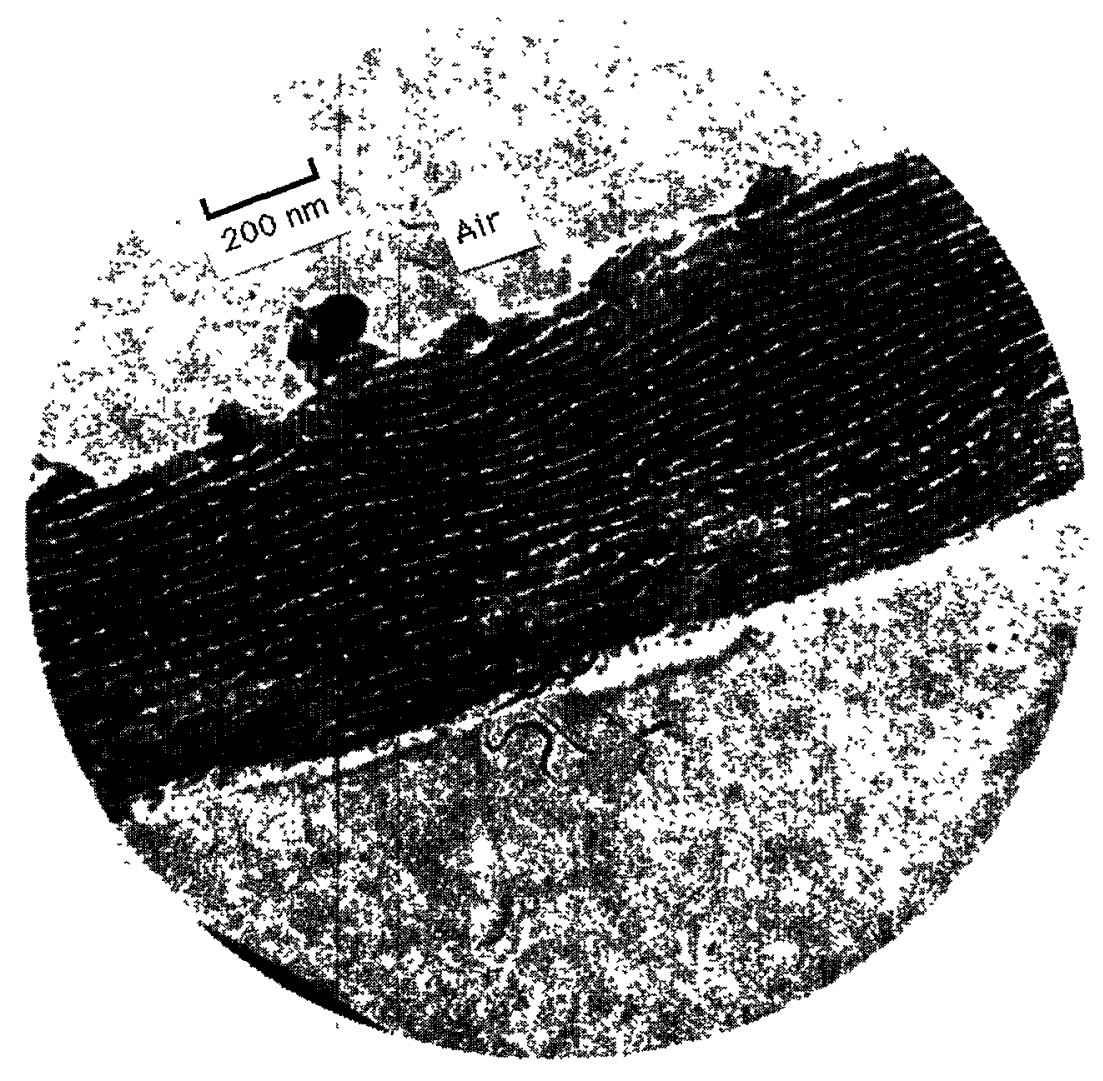

Fig. 1. - (Contrnued)

The lamellar structure appears weakened. For slightly higher nanoparticle volume fractions, we would expect a nanoparticle-induced transition towards cylindrical domains. Indeed, the presence of the nanoparticles induces an additionnal stretching of only one of the two blocks of the copolymer chain and this asymmetric stretching leads to a spontaneous curvature of the A-B interface towards the block which is the less stretched. An intermediate system in between the lamellar and cylindrical structures has actually been obtained with lower volume fractions, but larger particles [11].

Figures $2 \mathrm{a}$ and $2 \mathrm{~b}-\mathrm{c}$ show optical micrographs of the relief domains obtained at the free surfaces of a pure symmetric copolymer film (2a) and a $25 \%$ composite with a symmetric copolymer matrix $(2 \mathrm{~b}, \mathrm{c})$. The annealing time was 36 hours at $T=150^{\circ} \mathrm{C}$ in all cases. Figure $2 a$ presents the population of polydisperse circular domains commonly observed in such films. The domains correspond to holes with a depth of one layer. By comparison, Figure $2 b$ exhibits anomalous domain shapes in the case of low domain density. Figure 2c corresponds to a higher domain density. In addition to the anomalous shape, it also shows a surprising monodispersity in the size distribution and a tendency towards ordering the domains in an hexagonal array. Since similar domain shape and organization have never been observed in pure copolymer systems, they have to be attributed to the presence of the particles in the lamellar structure. Fluctuating domain shapes in Figure $2 \mathrm{~b}$ imply a reduction in the effective 
a)

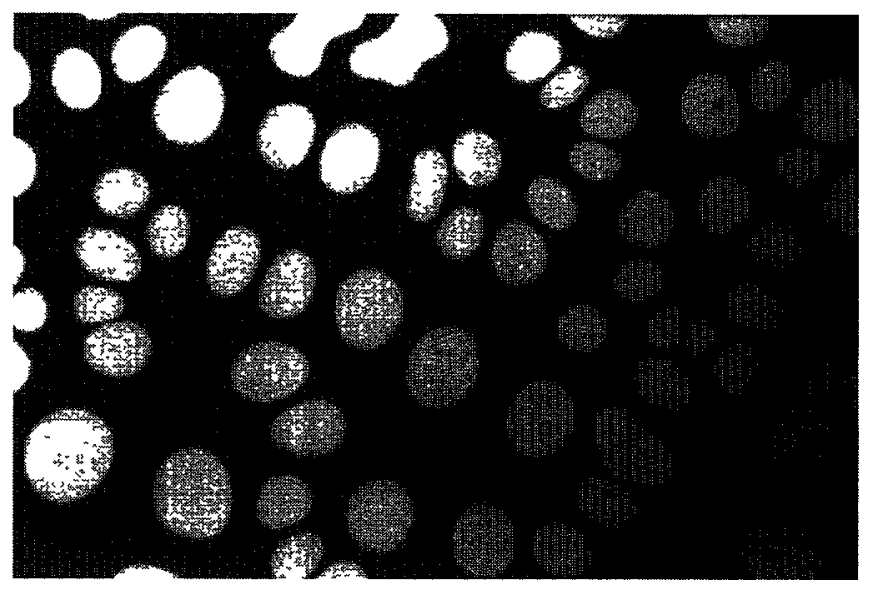

b)
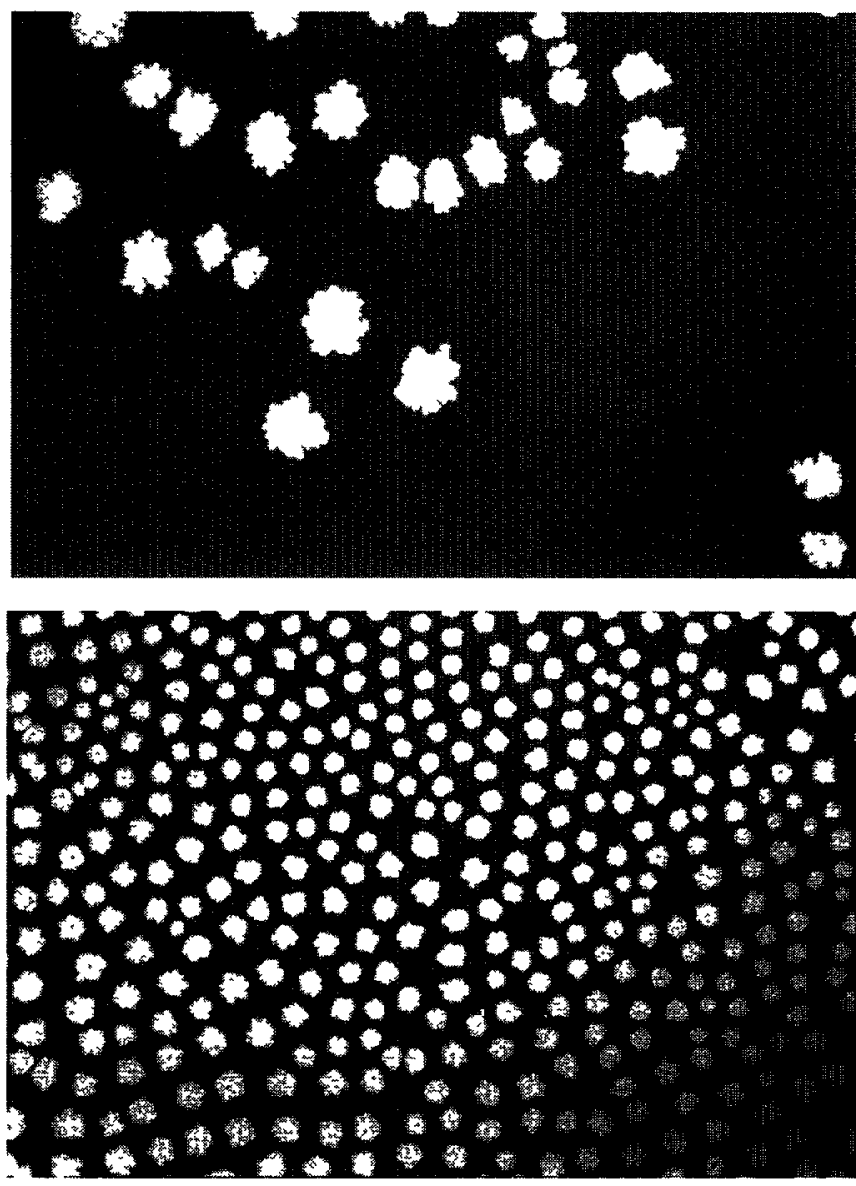

Fig. 2. - a) Optical micrograph (in reflexion) of the free surface of a symmetric pure copolymer film. Full size: 190 micrometers. White spots are depressions a depth of about $30 \mathrm{~nm}$. b, c) Optical micrograph (in reflexion) of the free surface of a composite symmetric copolymer film. Full size: 190 micrometers. Bright areas are depressions with a depth of about $32 \mathrm{~nm}$. Figures $2 \mathrm{~b}$ and $2 \mathrm{c}$ differ by the density of holes, controlled by the total film thickness. 
line tension $\tau$ at the domain edge, with respect to that of pure copolymer systems. Since the bilayer step at the edge necessarily corresponds to a defect line in the lamellar structure (dislocation [12] or else [13]), one could reasonably explain the decrease of $\tau$ by a relaxation of local elastic stresses due to an excess amount of nanoparticles in the defect region. The anomalous features of the domain population are more puzzling. The peaking of the domain size distribution and the correlations in the domain positions suggest a cooperative mechanism during the domain nucleation stage itself. Long range interactions induced by the presence of the nano particles can be invoked. They would be mediated by long range elastic stresses in the organized composite. However such exploratory digressions ignore an important dimension of our system, which is the magnetic character of the particles. Although the behaviour of superparamagnetic particles is expected to be insensitive to particle interactions, even at high concentrations, we cannot totally exclude the possibility of a magnetic origin in the ordering. Some of the nanoparticle induced modifications of the domain shapes could therefore also be attributed to long range dipolar interactions similar to those reported in other bidimensional systems [14].

Figure 3 has two goals. The first one is to demonstrate the role of the polymer layer grafted at the surface of the nanoparticles. For that purpose, the polymer layer has now been replaced by a layer of a commercial surfactant (BEYCOSTAT NE, CECCA), which is efficient enough to get a stable colloïdal solution in toluene. The average diameter of the particles is also appreciably larger than before, about $10 \mathrm{~nm}$, and their volume fraction in the composite is $0.1 \%$. The second goal is to illustrate the correlation between composite structure and thin film surface topography. Figure 3a shows a TEM cross section of the film. The nanoparticles clearly concentrate at the solid/film and film/air interfaces rather than inside the copolymer film. Moreover, the way they are ejected from the mixture is spectacular; a local disorientation of the lamellae of the structure provides vertical channels for particle ejection. This leads to the formation of a sort of microscopic volcano, which expulses nanoparticles. Figure $3 \mathrm{~b}$ presents an AFM picture of the same composite film. It exhibits characteristic elevations, irregular in shape and size, which correspond to the same volcanoes.

One point needs to be clarified. When going from the particles of Figures 3 to those of Figure 1, two parameters have been changed: their size and the chemical nature of the protecting layer. The specific role of the polymer layer on compatibility cannot be demonstrated in that way. Preliminary experiments, performed with large PS-covered nanoparticles, show that the compatibility is then restored, but different structures appear.

To conclude, we have demonstrated the possibility of elaborating highly organized stable organic-inorganic compounds by mixing nanoparticles and diblock copolymers. Thin films of these composite materials exhibit original macroscopic features, which still remain to be explored. The simultaneous use of AFM and TEM techniques allowed us to correlate surface features and material behaviour, which opens up a simple and fast route for systematic investigations.

\section{Acknowledgments}

We thank P. Lutz for providing us with the short PS chains with a sulfonated termination and C. Williams for critical relecture of the manuscript and numerous improvements in the text. 
a)
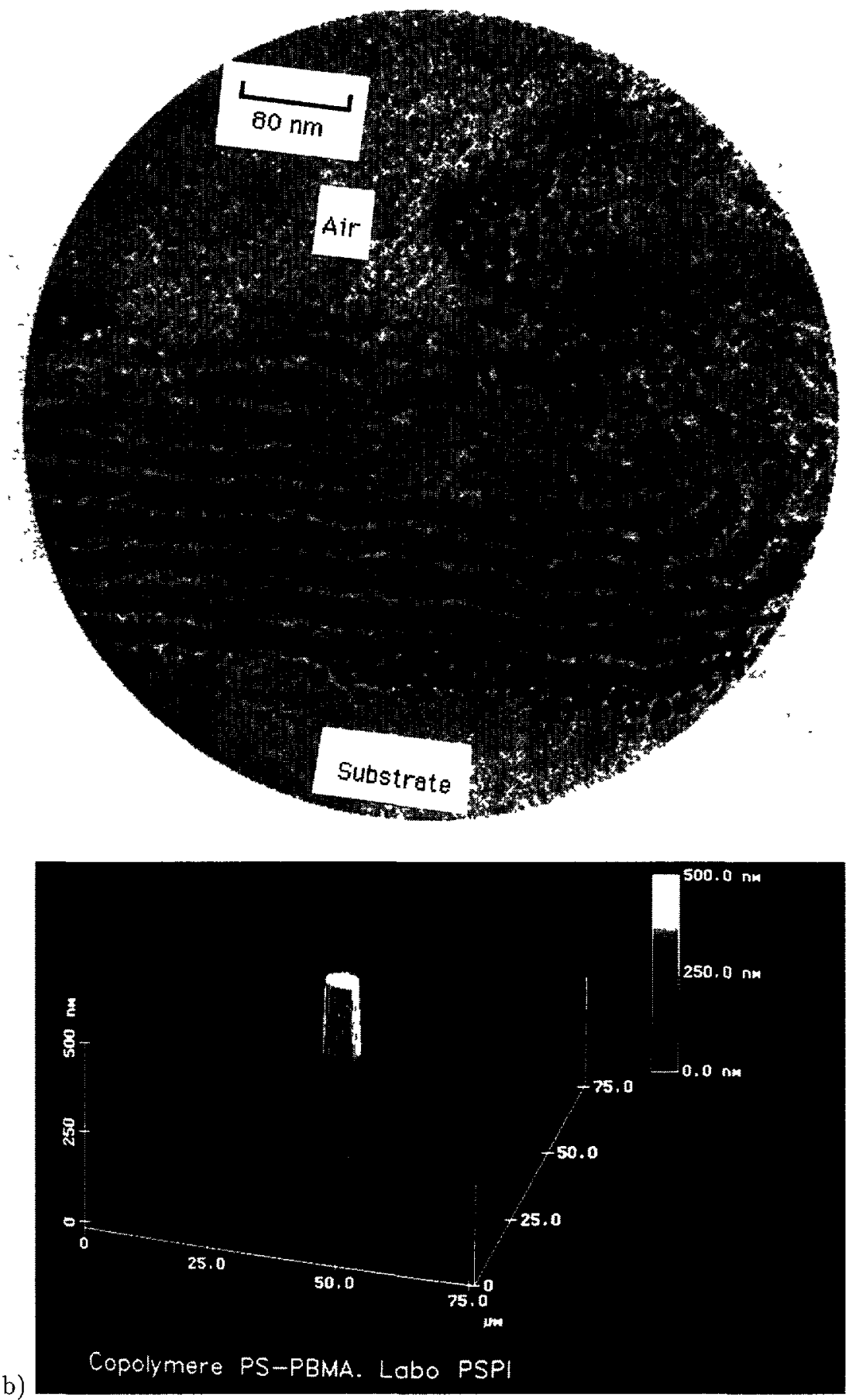

Fig. 3. - a) TEM micrograph of the cross-section of a composite film. A surfactant layer is grafted on the particles. Particle diameter $=7 \mathrm{~nm}$. b) AFM view of the free surface of the same film. 


\section{References}

[1] Ajdari A. and Leibler L., La Recherche 223 (1991) 732.

[2] Henkee C.S., Thomas E.L. and Fetters L.J., J. Mater. Sci 23 (1988) 1685.

[3] Ng Cheong Y., Schrock R.R., Cohen R.E., J. Chem. Mater. 4 (1992) 24.

[4] Saito R., Okamura S. and Ishizu K., Polymer 33 (1992) 1099.

[5] Saito R., Okamura S. and Ishizu K., Polymer 34 (1993) 1183.

[6] Tannenbaum R., in "Short and long chains at interfaces", Series: Moriond Condensed Matter Physics (ed. Frontières, Gif-sur-Yvette, France, 1995) pp. 95-100.

[7] Coulon G., Ausserré D. and Russell T.P., Polym. Prep., ACS Polym. div. 30 (1989) 366; Coulon G., Ausserré D. and Russell T.P., J. Phys. France 51 (1990) 777.

[8] Bacri J.C., Perzynski R., Salin D., Cabuil V. and Massart R., J. Magn. Magn. Mater. 62 (1986) 36.

[9] Cabuil V., Hochart N., Perzynski R. and Lutz P.J., Progr. Colloid. Polymer Sci. 97(1994) 71.

[10] Trent J.S., Scheinbeim J.I. and Couchman P.R., Macromolecules 16 (1983) 589.

[11] Hamdoun B., Thèse de Doctorat de l'Université du Maine, Le Mans, France (1995).

[12] Turner M.S., Maaloum M., Ausserré D., Joanny J.F. and Kunz M., J. Phys. II France 4 (1994) 689.

[13] Carvalho B.L. and Thomas E.L., Phys. Rev. Lett. 73 (1994) 3321.

[14] Seul M. and Andelman D., Science 267 (1995) 476. 